\title{
Factores asociados al riesgo de mediastinitis postoperatoria en cirugía cardiovascular en el Instituto Nacional del Corazón (INCOR)
}

\author{
Associated risk factors for mediastinitis post cardiovascular surgery in the Instituto Nacional del Corazón \\ (INCOR)
}

\section{Sr. Editor:}

La mediastinitis es una importante complicación de los procedimientos quirúrgicos cardiovasculares que tiene consecuencias posteriores para el paciente y que en ocasiones requiere re-exploración quirúrgica para el diagnóstico y siempre para el tratamiento (1$10)$.

Por medio de esta carta, hacemos referencia a un estudio realizado en el Instituto Nacional del Corazón de la Seguridad Social de Lima - Perú (INCOR), que tuvo como objetivos identificar las variables asociadas al desarrollo de mediastinitis en los pacientes sometidos a cirugía cardiaca, identificar los principales factores de riesgo pre, intra y post operatorios asociados con mediastinitis, y categorizar los factores de riesgo según prioridad y medir la fuerza de asociación de cada uno de los factores de riesgo.

El estudio fue de casos y controles, con una confianza de $95 \%$, una potencia del $80 \%$, una tasa prevista de factores asociados al riesgo de $50 \%$ para los controles, y un odds ratio (OR) de 3 para encontrar diferencias. Se estudiaron 45 casos y 90 controles de pacientes adultos sometidos a cirugía cardíaca entre el 1 enero 1996 al 31 de diciembre del 2000. Se determinó OR y realizó análisis multivariado.

Se encontró mediastinitis post operatoria en $45 / 2001(2,25 \%)$ casos y la mortalidad atribuida fue $2,2 \%$. El clampaje de aorta $>2$ horas, la presencia de infarto agudo de miocardio (IMA) antes de la cirugía y la fracción de eyección (FE) $<35 \%$ fueron factores independientes predictores de mediastinitis. La obesidad incrementó el riesgo 2,9 veces. El germen más común fue el Staphylococcus aureus (51,3\%).

Se concluyó que la mediastinitis post-operatoria es un evento multifactorial relacionado a la característica antropométrica del huésped, al infarto miocárdico pre operatorio y la baja FE.

A pesar del tiempo de realización del trabajo, creemos que es importante el conocimiento de nuestro estudio dado que nuestros resultados pueden tener valor comparativo con otros trabajos en el tiempo.

$$
\text { José Merino }{ }^{1, a} \text {, Javier Cieza }{ }^{2,3, b}
$$

\section{REFERENCIAS BIBLIOGRÁFICAS}

1. Bosen D, Mackavich S. Tratamiento de la mediastinitis tras cirugía cardíaca. Nursing. 2007; 25 (7):39-42

2. Careaga G, Campos R. Tratamiento de la mediastinitis y otras complicaciones de la esternotomía en cirugía cardiaca. Cir Gen. 2010; 32(4):217-220.

3. Diez C, Koch D, Kuss O, Silber R, Friedrich I, Boergermann J. Risk factors for mediastinitis after cardiac surgery: a retrospective analysis of 1700 patients. J Cardiothorac Surg. 2007; 2:23-30. doi: 10.1186/1749-8090-2-23

4. Eklund AM, Lyytikäinen $O$, Klemets $P$, et al. Mediastinitis after more than 10,000 cardiac surgical

1. Servicio de Cirugía de Tórax Cardiovascular, Hospital Nacional Arzobispo Loayza. Lima, Perú.

2. Facultad de Medicina, Universidad Peruana Cayetano Heredia. Lima, Perú.

3. Servicio de Nefrología, Hospital Nacional Cayetano Heredia, Lima, Perú.

a. Magister en Medicina;

b. Doctor en Medicina 
procedures. Ann Thorac Surg. 2006; 82:1784-1789.

5. Fernández-de-Larrea $N$, Martín $M$, Maeso $S$, et al. Mediastinitis tras cirugía cardíaca: estudio de casos y controles para detectar áreas de mejora. Rev Calidad Asistencial. 2006; 21(6):281-6.

6. Fernández-Palacios JA. Mediastinitis postesternotomia media en cirugía cardiaca. Span J Surg Res. 2008; 11(4):149-152.

7. López MJ, San Juan R, Aguado JM, et al. Casecontrol study of risk factors for mediastinitis after cardiovascular surgery. Infect Control Hosp Epidemiol. 2006; 27(12):1397-400.

8. Llanes J, Pérez H, Rodríguez L, et al. Influencia de algunos factores de riesgo cardiovasculares sobre los eventos clínicos adversos más frecuentes, en pacientes revascularizados con circulación extracorpórea. Rev Cubana Cir. 2011; 50(4):462-471.
9. Robinson PJ, Billah B, Leder K, Reid CM, ASCTS Database Committee. Factors associated with deep sternal wound infection and haemorrhage following cardiac surgery in Victoria. Interact Cardiovasc Thorac Surg. 2007; 6(2):167-71.

10. Toumpoulis IK1, Theakos N, Dunning J. Does bilateral internal thoracic artery harvest increase the risk of mediastinitis? Interact Cardiovasc Thorac Surg. 2007; 6(6):787-91.

Recibido: 13/11/2019

Aceptado: 28/12/2019 